Supporting Information for:

\title{
Stepwise Two-Photon-Induced Fast Photoswitching via Electron Transfer in Higher Excited States of Photochromic Imidazole Dimer
}

Yoichi Kobayashi ${ }^{\dagger}$, Tetsuro Katayama ${ }^{\star}$, Takuya Yamane ${ }^{\dagger}$, Kenji Setoura ${ }^{\ddagger}$, Syoji

Ito $^{\ddagger}$, Hiroshi Miyasaka ${ }^{*}$, and Jiro Abe ${ }^{*}, \dagger$

${ }^{\dagger}$ Department of Chemistry, School of Science and Engineering, Aoyama Gakuin University, 5-10-1

Fuchinobe, Chuo-ku, Sagamihara, Kanagawa 252-5258, Japan

${ }^{7}$ Division of Frontier Materials Science and Center for Promotion of Advanced Interdisciplinary Research,

Graduate School of Engineering Science, Osaka University, Toyonaka, Osaka 560-8531, Japan

E-mail:jiro_abe@chem.aoyama.ac.jp,miyasaka@chem.es.osaka-u.ac.jp

\section{CONTENTS}

1. ${ }^{1}$ H NMR Spectra $\quad$ S2

2. HR-ESI-TOF-MS-Spectra $\quad$ S4

3. HPLC Chromatograms S6

$\begin{array}{ll}\text { 4. LC/MS Spectra } & \text { S7 }\end{array}$

5. Picosecond Transient Absorption Spectra of ZnTPP S8

6. Emission Decay Profiles of 1 and ZnTPP $\quad$ S9

7. Transient Absorption Dynamics of 1 with a Picosecond Laser Pulse S10

8. Estimation of the Charge Separated State between ZnTPP and ImD S11

9. Comparison of the Transient Absorption Spectra of 1 at Millisecond Time Scales $\quad \mathrm{S12}$

10. Kinetics for the Thermal Back Reaction $\quad$ S12

11. Pump Power Dependence of the Transient Absorption Signal of 1 $\begin{array}{lr}\text { by Using Nanosecond Laser Pulses } & \text { S14 }\end{array}$

12. DFT Calculations $\quad$ S15

13. References $\quad$ S21 


\section{1. ${ }^{1}$ H NMR Spectra}

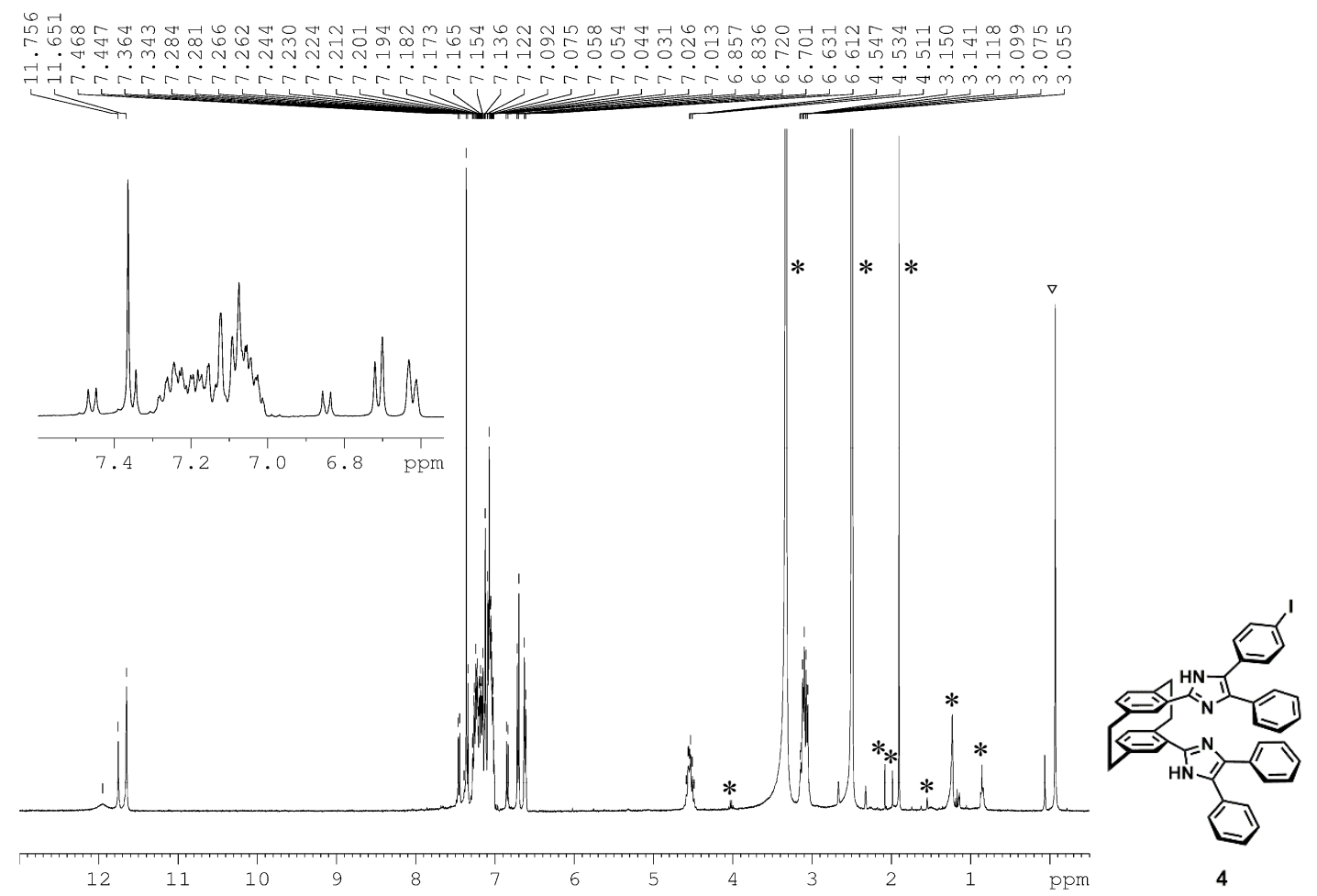

Figure S1. ${ }^{1} \mathrm{H}$ NMR spectrum of compound 4 in DMSO- $d_{6}(*$ solvent peaks and $\nabla$ silicone grease $)$.

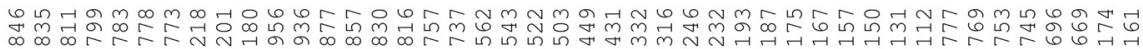

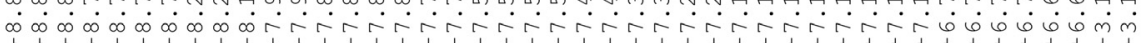

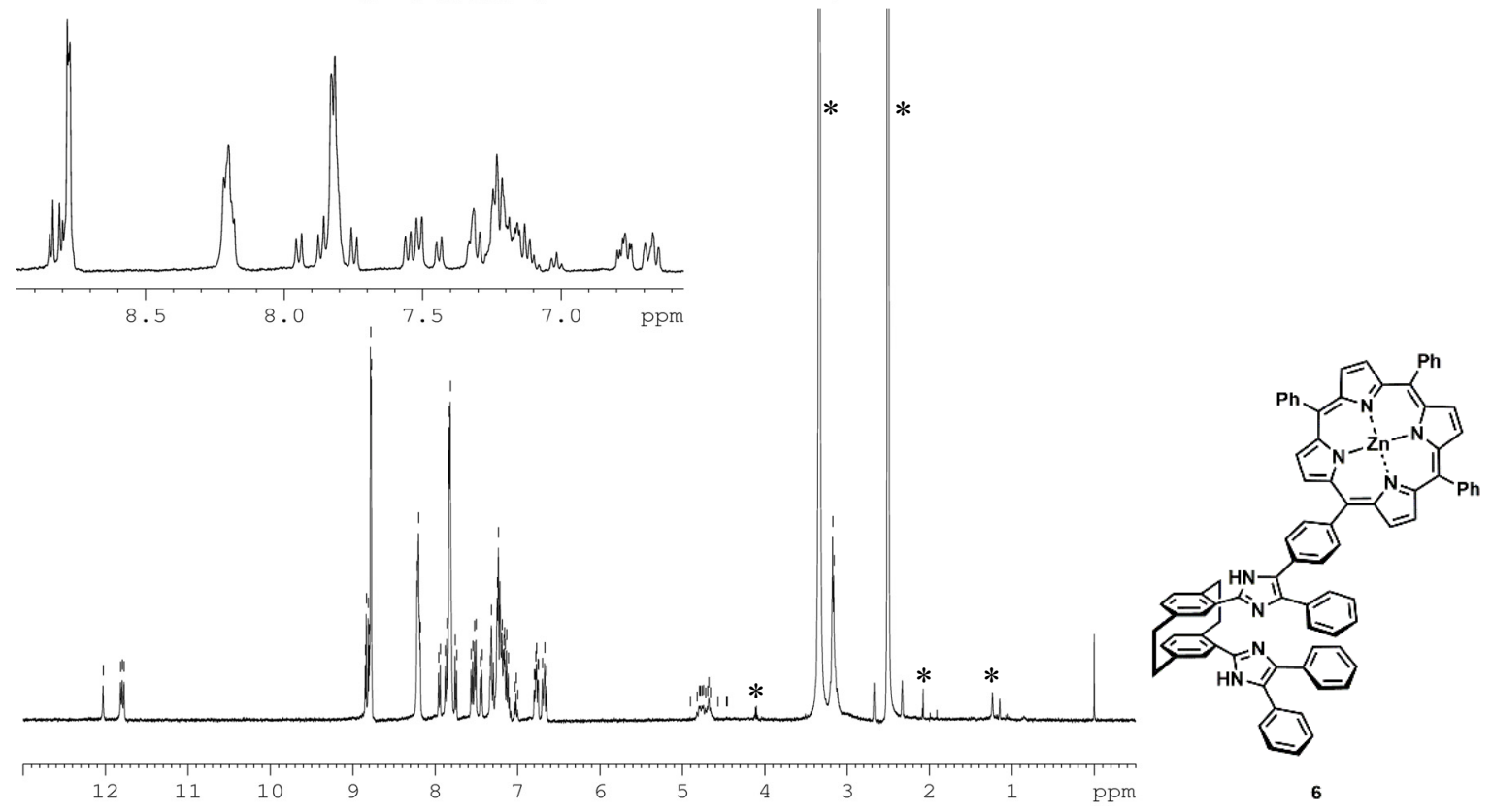

Figure S2. ${ }^{1} \mathrm{H}$ NMR spectrum of compound 6 in DMSO- $d_{6}(*$ solvent peaks). 

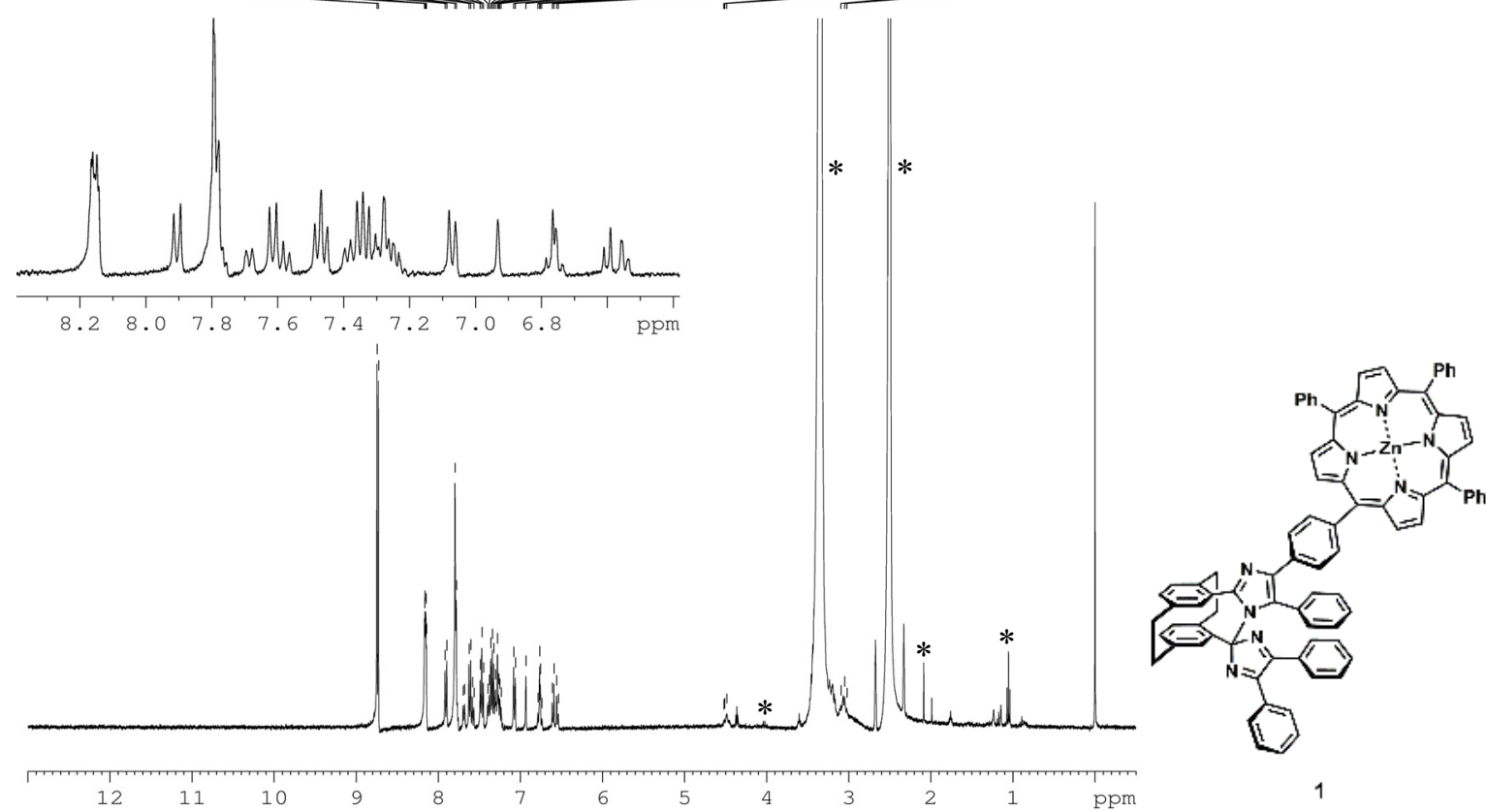

Figure S3. ${ }^{1} \mathrm{H}$ NMR spectrum of compound $\mathbf{1}$ in DMSO- $d_{6}$ (* solvent peaks). 


\section{HR-ESI-TOF-MS-Spectra}

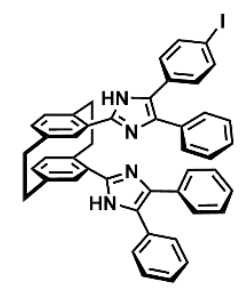

4

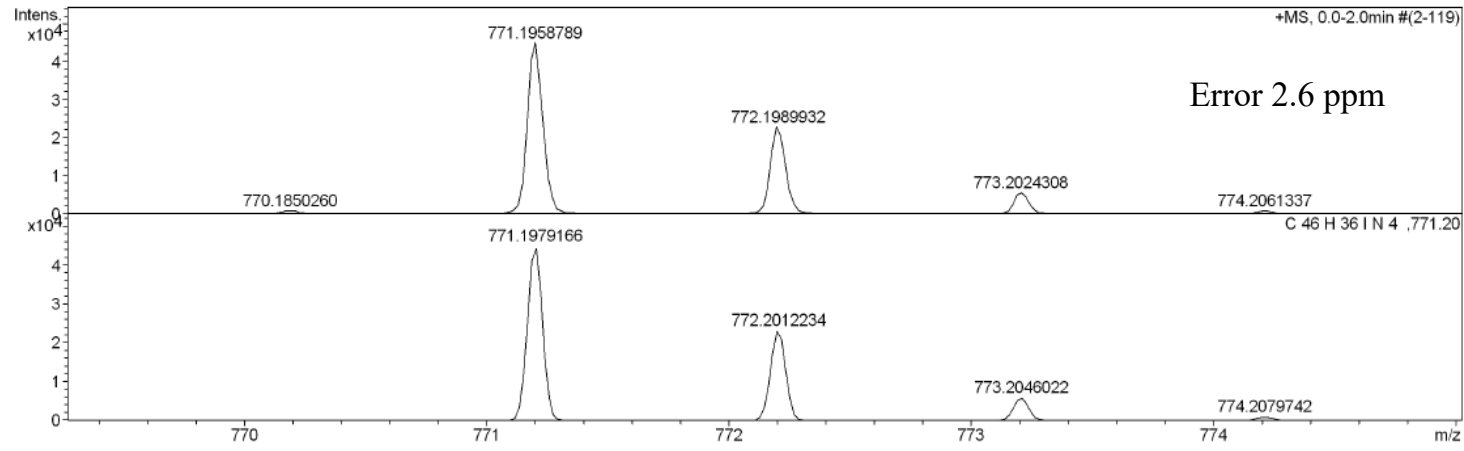

Figure S4 HR-ESI-TOF-MS of compound 4.

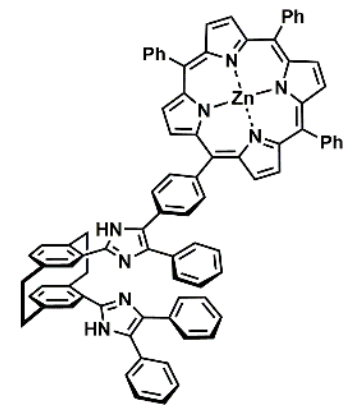

6

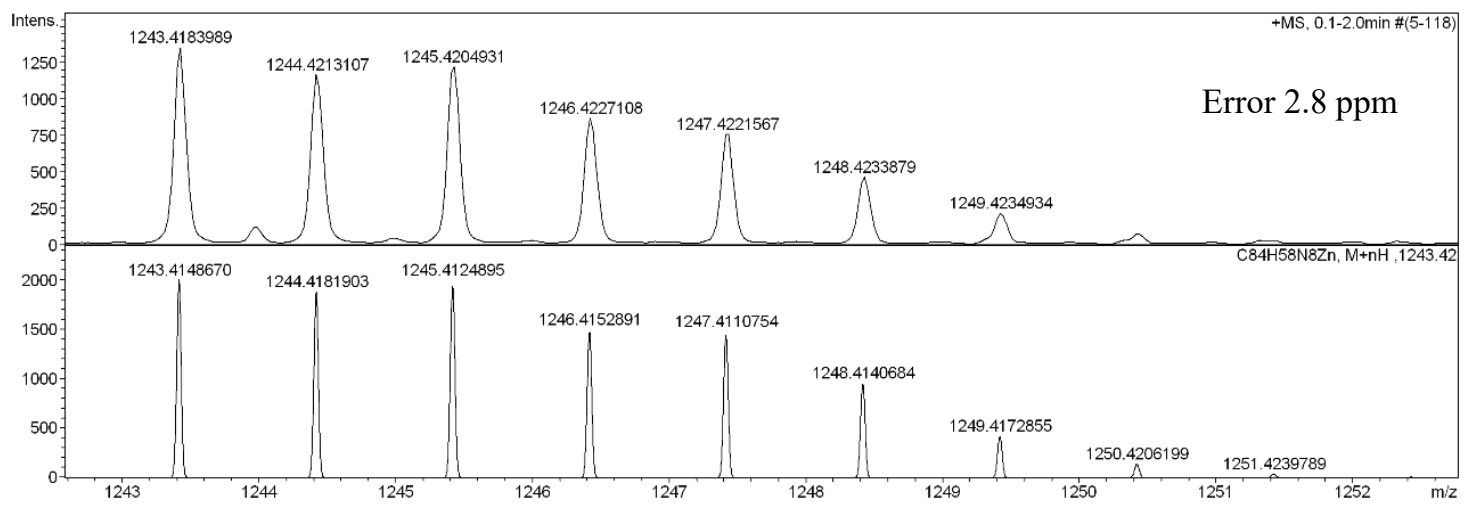

Figure S5 HR-ESI-TOF-MS of compound 6. 

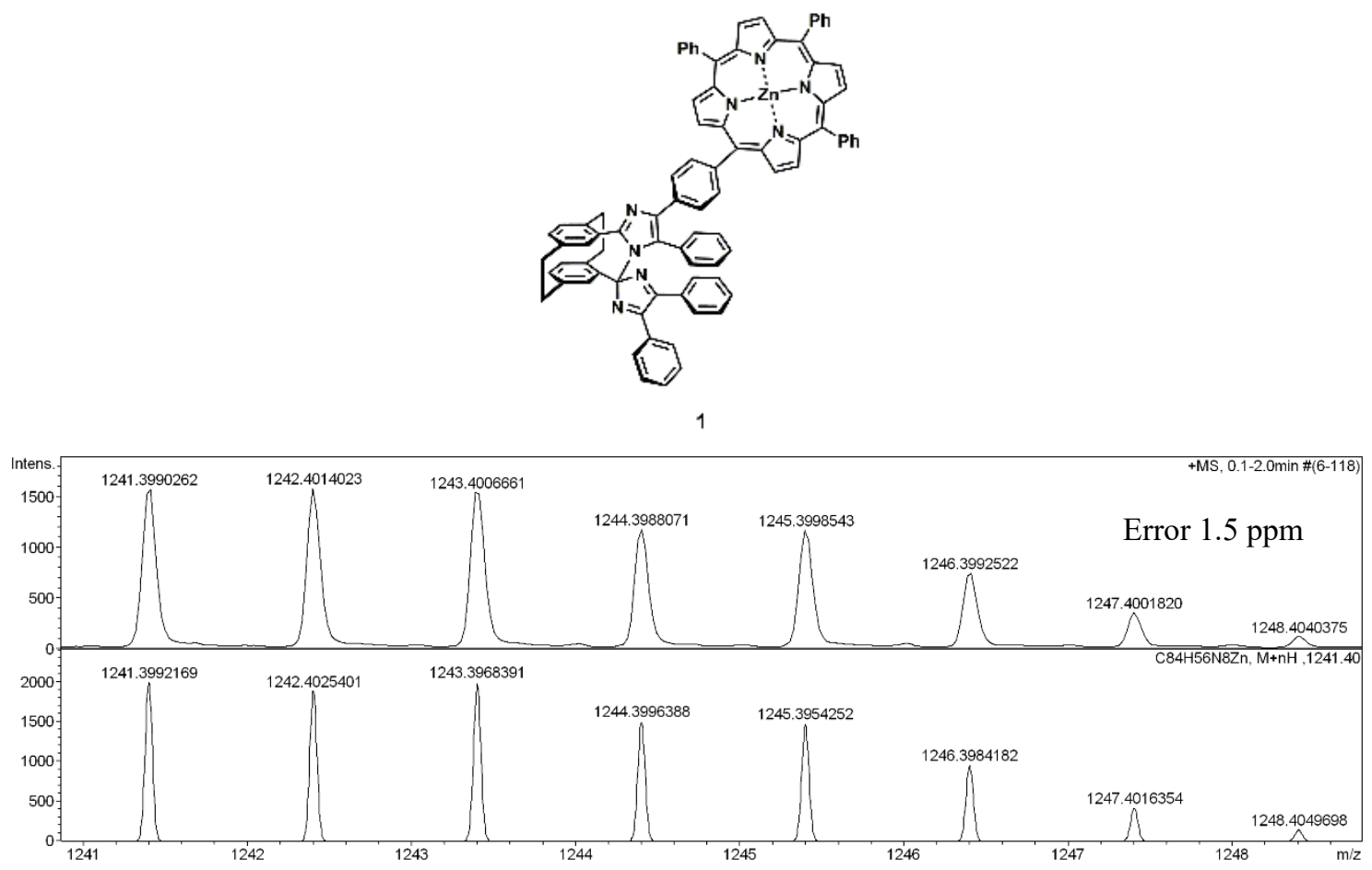

Figure S6 HR-ESI-TOF-MS of compound 1. 


\section{HPLC Chromatograms}

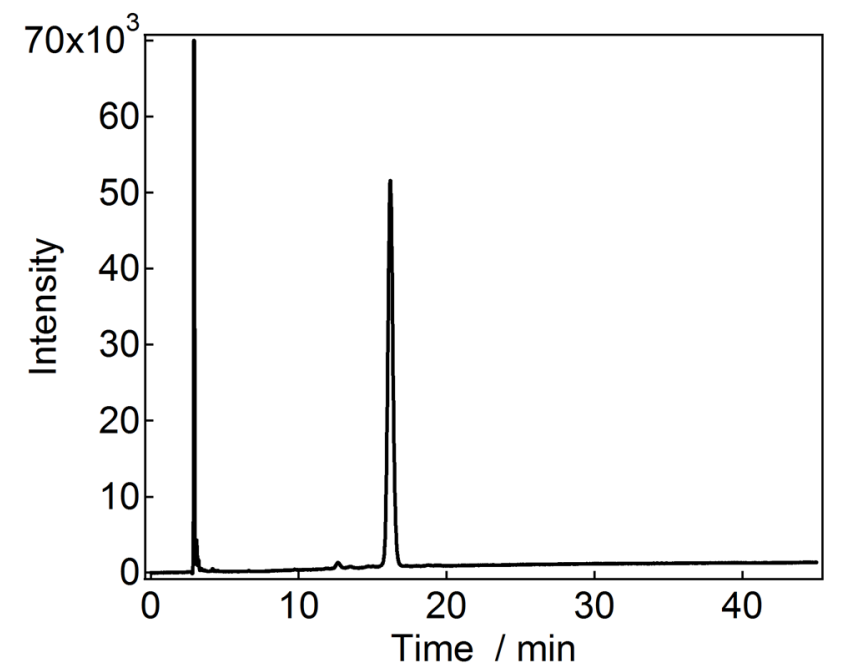

Figure S7. HPLC chromatogram of compound 1;98\% purity. HPLC analysis was performed using a reverse phase analytical column (Mightysil RP18, $25 \mathrm{~cm} \times 4.6 \mathrm{~mm}, 5 \mu \mathrm{m}$ particle) from Kanto Chemical Industries, equipped with a UV detector; the mobile phase was $\mathrm{MeOH}: \mathrm{CH}_{3} \mathrm{CN}=3: 1$ with a flow rate of $1.0 \mathrm{~mL} / \mathrm{min}$, detection wavelength; $254 \mathrm{~nm}$.

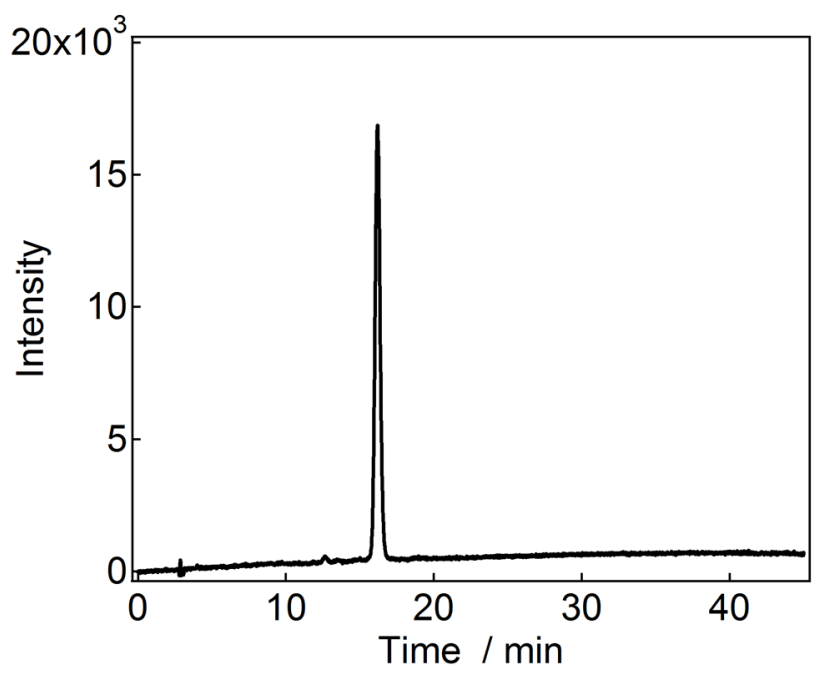

Figure S8. HPLC chromatogram of compound 1; 98\% purity. HPLC analysis was performed using a reverse phase analytical column (Mightysil RP18, $25 \mathrm{~cm} \times 4.6 \mathrm{~mm}, 5 \mu \mathrm{m}$ particle) from Kanto Chemical Industries, equipped with a UV detector; the mobile phase was $\mathrm{MeOH}: \mathrm{CH}_{3} \mathrm{CN}=3: 1$ with a flow rate of $1.0 \mathrm{~mL} / \mathrm{min}$, detection wavelength; $365 \mathrm{~nm}$. 


\section{LC/MS Spectra}

Figure S9 shows Liquid chromatography-mass spectrometry (LC/MS) charts of 1 (eluent : $\mathrm{MeOH}: \mathrm{CH}_{3} \mathrm{CN}=3: 1$ ) (a) before and (b) after $365 \mathrm{~nm}$ continuous wave UV light irradiation for 10 seconds. The peak corresponding to 1 was separated into 4 peaks with the same molar mass after UV light irradiation. This experiment show that different structural isomers of $\mathbf{1}$ are formed once UV light is irradiated.

(a)

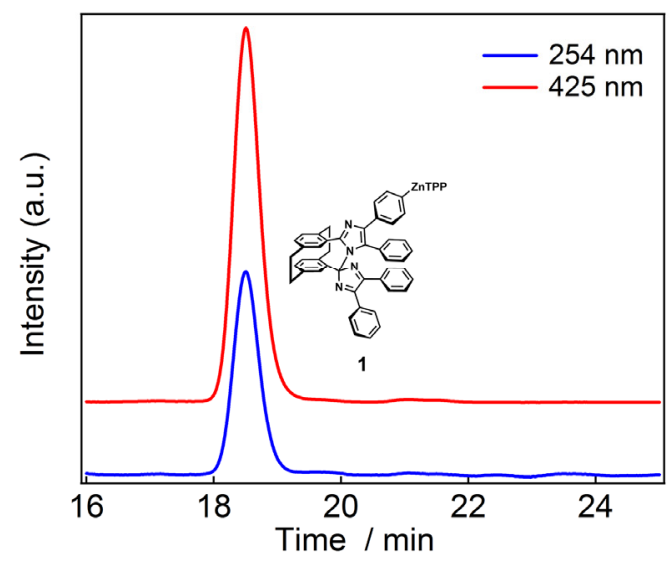

(b)

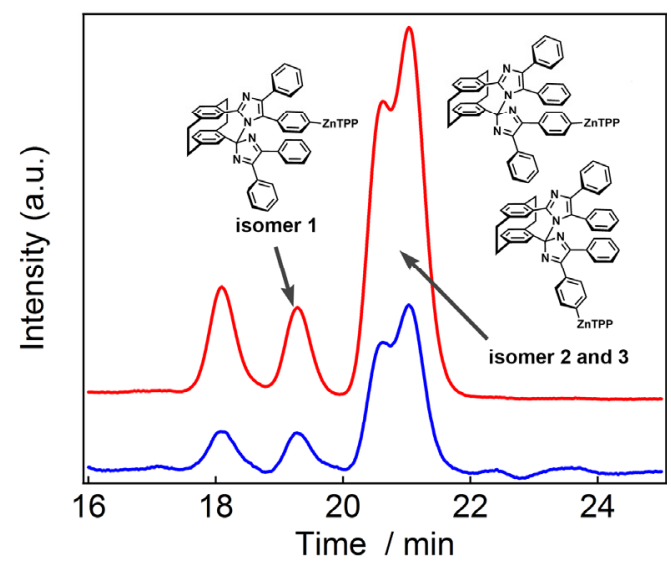

Figure S9. LC/MS charts of 1 (eluent : $\mathrm{MeOH}: \mathrm{CH}_{3} \mathrm{CN}=3: 1$ ) (a) before and (b) after $365 \mathrm{~nm}$ continuous wave UV light irradiation for 10 seconds. 


\section{Picosecond Transient Absorption Spectra of ZnTPP}

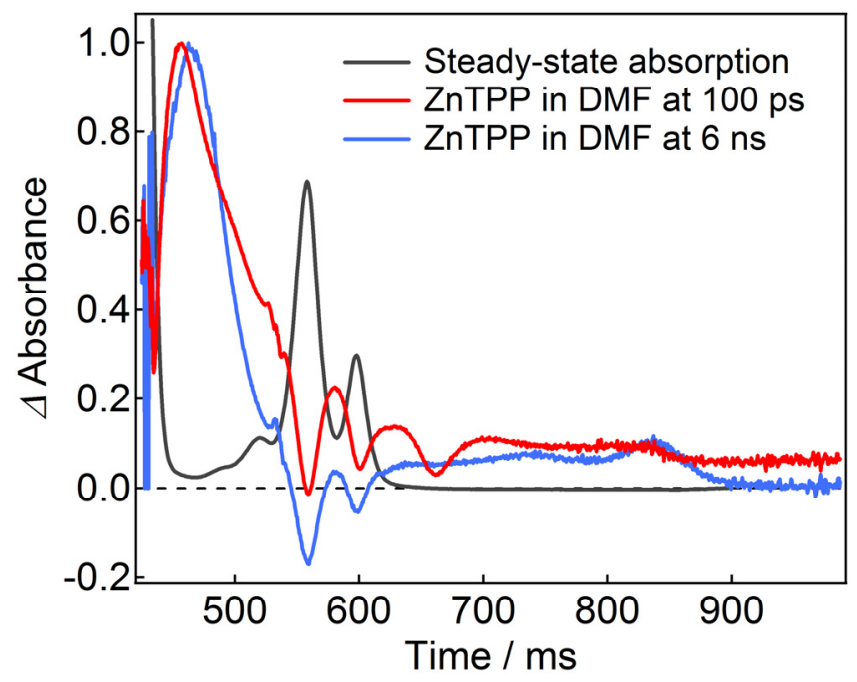

Figure S10. Steady-state absorption (gray line) and transient absorption spectra of ZnTPP in N,Ndimethylformamide (DMF) at $100 \mathrm{ps}$ (red line) and $6 \mathrm{~ns}$ (blue line) after the picosecond pulse excitation at $532 \mathrm{~nm}$ (transient absorption signals are normalized) . 


\section{Emission Decay Profiles of 1 and ZnTPP}

The excitation and probe wavelengths were set to $546 \mathrm{~nm}$ and $600 \mathrm{~nm}$, respectively. Figure S9 shows the emission decay profiles of 1 and $\mathrm{ZnTPP}$ in benzene $\left(8.1 \times 10^{-6} \mathrm{M}\right)$. The IRF is shown as a gray line. The emission decay of ZnTPP follows the single exponential decay kinetics and the half-life is $1.9 \mathrm{~ns}$. On the other hand, the emission decay of $\mathbf{1}$ is completely the same as that of ZnTPP. This result clearly shows that there is no additional relaxation pathway from the $\mathrm{S}_{1}$ state of the ZnTPP moiety in $\mathbf{1}$ as compared to that of ZnTPP. That is, one photon process does not contribute to the photochromism of $\mathbf{1}$ and only the two-photon process can induce photochromism by visible light.

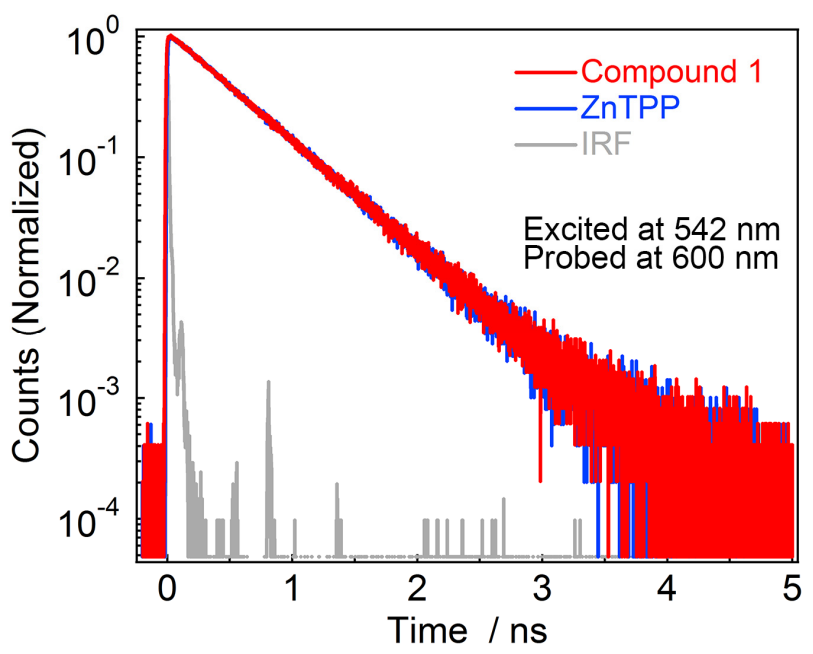

Figure S11. Emission decay profiles of 1 and ZnTPP in benzene $\left(8.1 \times 10^{-6} \mathrm{M}\right)$ excited and probed at 546 and $600 \mathrm{~nm}$, respectively. A gray line shows the instrumental response function (IRF). 


\section{Transient Absorption Dynamics of 1 with a Picosecond Laser Pulse}

Transient absorption dynamics of $\mathbf{1}$ excited at $532 \mathrm{~nm}$ of a ps laser pulse and probed at $460 \mathrm{~nm}$ is shown in Figure S10. These dynamics were fitted with single and double exponential decay functions convoluted by a Gaussian function for dynamics excited at low $\left(0.06 \mathrm{~mJ} \mathrm{~mm} \mathrm{~mm}^{-2}\right)$ and high $\left(1.2 \mathrm{~mJ} \mathrm{~mm}^{-2}\right)$ pump powers, respectively. The IRF for dynamics excited at low and high pump powers were set to be 16 and $20 \mathrm{ps,} \mathrm{respectively.} \mathrm{The} \mathrm{time} \mathrm{constant} \mathrm{of} \mathrm{the} \mathrm{fast} \mathrm{decay}$ component of the dynamics excited at the high pump power is $26 \mathrm{ps}$.

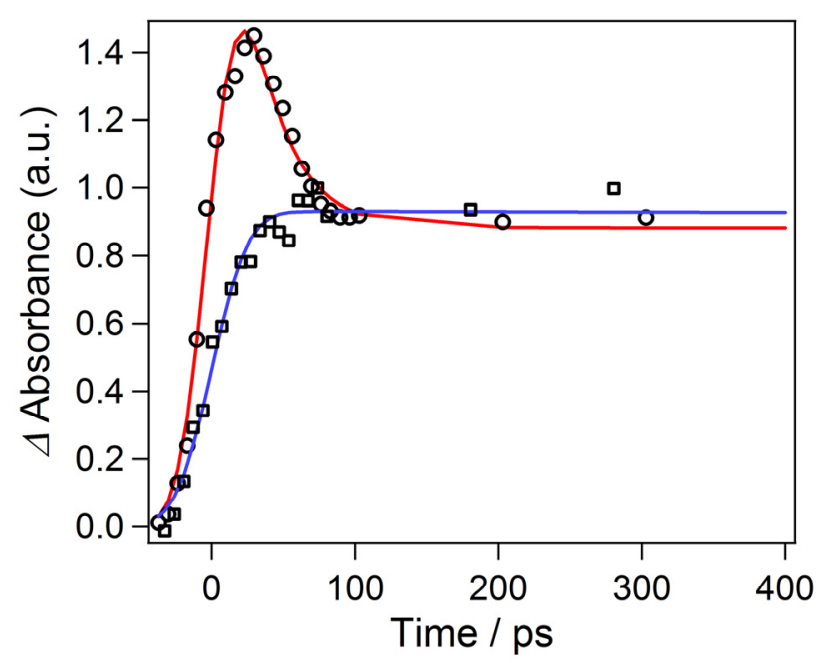

Figure S12. Transient absorption dynamics of 1 excited at $532 \mathrm{~nm}$ of a ps laser pulse and probed at $460 \mathrm{~nm}$. The dynamics excited at the low $\left(0.06 \mathrm{~mJ} \mathrm{~mm}^{-2}\right)$ and high $\left(1.2 \mathrm{~mJ} \mathrm{~mm}^{-2}\right)$ pump powers are shown in blue and red color, respectively. 


\section{Estimation of the Charge Separated State between ZnTPP and ImD}

The energy level of the CS state between the ZnTPP and ImD moieties in $\mathbf{1}$ was estimated on the basis of eq. $\mathrm{S} 1 .^{\mathrm{S} 1}$

$$
E_{C S}=E_{o x}-E_{r e d}-\frac{e^{2}}{4 \pi \varepsilon_{0} \varepsilon r_{C S}}
$$

Here, $E_{\mathrm{ox}}$ and $E_{\text {red }}$ are respectively the oxidation potential of the $\mathrm{ZnTPP}^{\mathrm{S} 2}$ moiety and the reduction potential of the $\operatorname{ImD}$ moiety ${ }^{\mathrm{S} 3}$. In the present estimation, the reduction potential of the unsubstituted $\mathrm{ImD}$ was used. The interionic distance, $r_{\mathrm{CS}}$, was set to be $1.14 \mathrm{~nm}$ as a center-tocenter distance between the two moieties on the basis of the geometry optimized by the DFT calculation (B3LYP/6-31G(d) level). $e, \varepsilon_{0}$, and $\varepsilon$ are respectively the elementary charge, dielectric constant of vacuum, and the relative dielectric constant of the medium.

The reduction and oxidation potentials measured in polar solutions such as acetonitrile or DMF were corrected for benzene solution by using the Born equation, eq S2.

$$
\Delta F_{S}=\frac{e^{2}}{8 \pi \varepsilon_{0}}\left(\frac{1}{R^{+}}+\frac{1}{R^{-}}\right)\left(\frac{1}{\varepsilon}-\frac{1}{\varepsilon_{R}}\right)
$$

$R^{+}$and $R^{-}$are the radius of the anion and that of the cation, respectively. In the actual estimation, the $R^{+}$and $R^{-}$values were respectively set to be 0.5 and $0.6 \mathrm{~nm} . \varepsilon_{\mathrm{R}}$ is the relative dielectric constant of the solvent employed for the electrochemical measurements and $\varepsilon$ is the relative dielectric constant of benzene, 2.28 .

The energy level of the CS state, $E_{\mathrm{CS}}$, thus estimated is $2.82 \mathrm{eV}$ and this value is higher than the $\mathrm{S}_{1}$ state of ZnTPP, $2.07 \mathrm{eV}$, and slightly lower than that of $\mathrm{ImD}, \sim 3.1 \mathrm{eV}$. 
9. Comparison of the Transient Absorption Spectra of 1 at Millisecond Time Scales

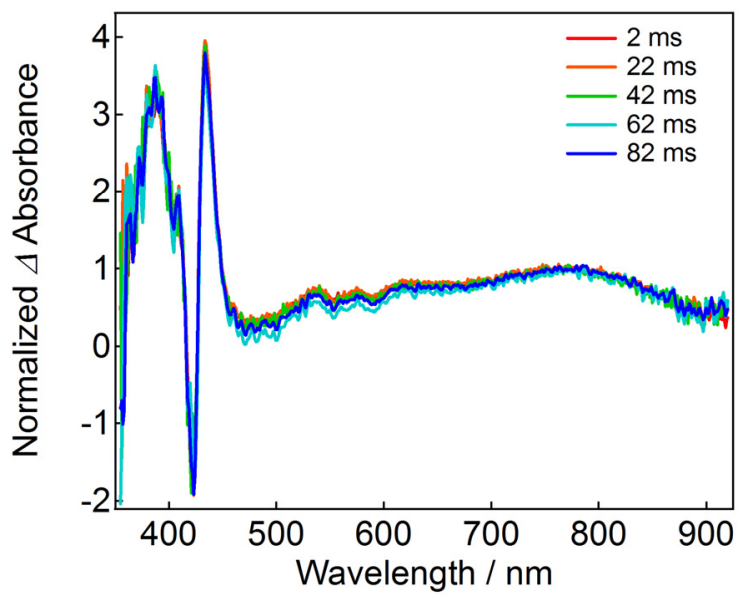

Figure S13. Normalized transient absorption spectra of 1 at different time delays in degassed benzene at $297 \mathrm{~K}\left(1.6 \times 10^{-6} \mathrm{M}\right)$ excited at $550 \mathrm{~nm}$ (pulse width $=\sim 5 \mathrm{~ns}$, intensity $\left.=11.6 \mathrm{~mJ}\right)$.

\section{Kinetics for the Thermal Back Reaction}

Table S1. First-order rate constants for the thermal back-reaction of $\mathbf{1 R}$.

\begin{tabular}{ccc}
\hline $\mathrm{T} / \mathrm{K}$ & $k / \mathrm{s}^{-1}$ & $\tau_{1 / 2} / \mathrm{ms}$ \\
\hline 278 & 2.98 & 233 \\
283 & 4.74 & 146 \\
288 & 7.45 & 93 \\
293 & 10.9 & 64 \\
298 & 18.4 & 38 \\
303 & 27.3 & 25 \\
308 & 40.6 & 17 \\
313 & 59.2 & 12 \\
\hline
\end{tabular}




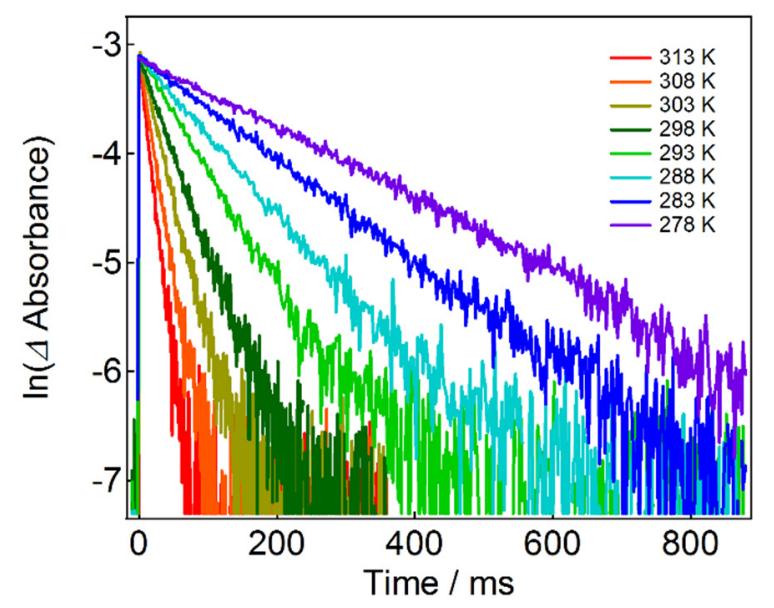

Figure S14. Transient absorption dynamics of 1 in degassed benzene $\left(5.0 \times 10^{-5} \mathrm{M}\right)$ excited at $355 \mathrm{~nm}$ (pulse width $=\sim 5 \mathrm{~ns}$, intensity $=4.0 \mathrm{~mJ}$ ) and probed at $760 \mathrm{~nm}$ at different temperature.

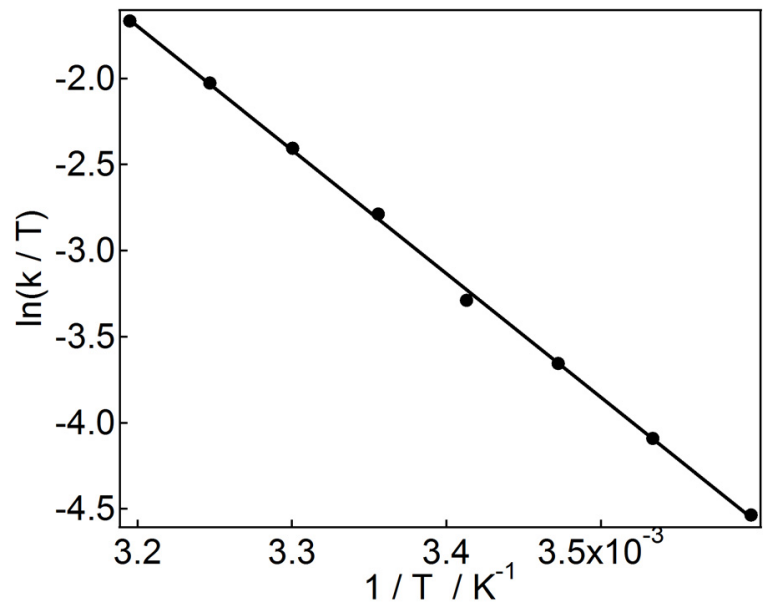

Figure S15. Eyring plots for the thermal back reaction of the radical species of $\mathbf{1}$ in degassed benzene $\left(5.0 \times 10^{-5} \mathrm{M}\right)$. 


\section{Pump Power Dependence of the Transient Absorption Signal of 1 by Using Nanosecond Laser Pulses}

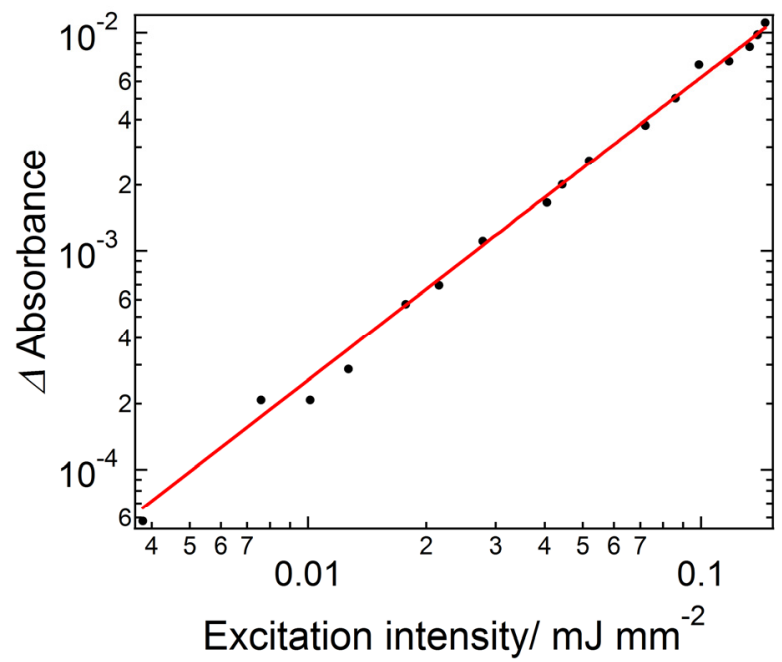

Figure S16. Pump power dependence of the logarithmic transient absorption signal of 1 in degassed benzene $\left(2.2 \times 10^{-5} \mathrm{M}\right.$ at $\left.298 \mathrm{~K}\right)$ excited at $550 \mathrm{~nm}$ and probed at $760 \mathrm{~nm}$. The slope of the fitting line is 1.4 . 


\section{DFT Calculations}

All calculations was carried out using the Gaussian 09 program (Revision D.01). ${ }^{\mathrm{S} 4}$ The molecular structure was fully optimized at the B3LYP/6-31G(d) level of theory, and analytical second derivative was computed using vibrational analysis to confirm each stationary point to be a minimum.

Table S2. Standard orientation of the optimized geometry for $\mathbf{1}$.

\begin{tabular}{|c|c|c|c|c|}
\hline \multirow{2}{*}{ Tag } & \multirow{2}{*}{ Symbol } & \multicolumn{3}{|c|}{ Coordinates (Angstroms) } \\
\hline & & $\mathrm{X}$ & $\mathrm{Y}$ & Z \\
\hline 1 & $\mathrm{C}$ & -9.0894190 & -5.5447720 & 1.2508310 \\
\hline 2 & $\mathrm{C}$ & -7.9755360 & -5.2859980 & 0.4469350 \\
\hline 3 & $\mathrm{C}$ & -9.7108270 & -4.4980220 & 1.9329460 \\
\hline 4 & $\mathrm{C}$ & -7.4858830 & -3.9886130 & 0.3278720 \\
\hline 5 & $\mathrm{C}$ & 5.6275440 & -7.4991470 & -2.0920020 \\
\hline 6 & $\mathrm{C}$ & 5.1652430 & -7.0892930 & -0.8400540 \\
\hline 7 & $\mathrm{C}$ & -9.2147900 & -3.1986010 & 1.8274850 \\
\hline 8 & $\mathrm{C}$ & -8.0934400 & -2.9308940 & 1.0260380 \\
\hline 9 & $\mathrm{C}$ & 6.1562710 & -6.5553740 & -2.9744730 \\
\hline 10 & $\mathrm{C}$ & 5.2312190 & -5.7443800 & -0.4733620 \\
\hline 11 & $\mathrm{C}$ & -7.5869570 & -1.5566810 & 0.8364190 \\
\hline 12 & $\mathrm{C}$ & -4.0762070 & -2.8167740 & 2.4029810 \\
\hline 13 & $\mathrm{C}$ & 6.2230640 & -5.2109520 & -2.6062820 \\
\hline 14 & $\mathrm{C}$ & -9.7291740 & 0.5401680 & -4.2244430 \\
\hline 15 & $\mathrm{C}$ & 5.7617030 & -4.7859250 & -1.3506890 \\
\hline 16 & $\mathrm{C}$ & -7.7111140 & -1.4762440 & 4.1074520 \\
\hline 17 & $\mathrm{C}$ & -8.0759670 & -1.4015100 & 5.4511510 \\
\hline 18 & $\mathrm{C}$ & -8.4932850 & 0.8217240 & -5.2214100 \\
\hline 19 & $\mathrm{C}$ & -4.2160050 & -2.0564130 & 1.2406290 \\
\hline 20 & $\mathrm{C}$ & -3.9679390 & -2.1840980 & 3.6433070 \\
\hline 21 & $\mathrm{C}$ & -8.6113980 & 0.3789710 & -1.9583760 \\
\hline 22 & $\mathrm{C}$ & -9.4938400 & 1.0453710 & -2.8149950 \\
\hline 23 & $\mathrm{C}$ & -7.5448890 & -0.4148010 & 1.8296150 \\
\hline 24 & $\mathrm{C}$ & -7.9621840 & -0.3960900 & 3.2461640 \\
\hline 25 & $\mathrm{C}$ & -8.7021090 & -0.2565840 & 5.9476020 \\
\hline 26 & $\mathrm{C}$ & 3.3249320 & -3.1870130 & -1.1758980 \\
\hline 27 & $\mathrm{C}$ & 8.3438230 & -3.5308420 & -0.8027950 \\
\hline
\end{tabular}




\begin{tabular}{|c|c|c|c|c|}
\hline 28 & $\mathrm{C}$ & 5.8326680 & -3.3413690 & -0.9560810 \\
\hline 29 & $\mathrm{C}$ & -6.8435290 & 0.2783560 & -0.1558870 \\
\hline 30 & $\mathrm{C}$ & -7.4459920 & 1.7322970 & -4.6174680 \\
\hline 31 & $\mathrm{C}$ & -4.2553170 & -0.6547020 & 1.3014090 \\
\hline 32 & $\mathrm{C}$ & -6.4688730 & 1.1880460 & -3.7752570 \\
\hline 33 & $\mathrm{C}$ & -7.9223450 & 1.0533960 & -0.9429940 \\
\hline 34 & $\mathrm{C}$ & -4.0074320 & -0.7896490 & 3.7162110 \\
\hline 35 & $\mathrm{C}$ & 7.1050760 & -2.7914270 & -0.7207940 \\
\hline 36 & $\mathrm{C}$ & 2.4120190 & -2.1949040 & -0.9927570 \\
\hline 37 & $\mathrm{C}$ & -9.9135460 & 2.3214710 & -2.4225630 \\
\hline 38 & $\mathrm{C}$ & 4.6210600 & -2.6333620 & -0.8588870 \\
\hline 39 & $\mathrm{C}$ & 9.3481020 & -2.6598780 & -0.5128930 \\
\hline 40 & $\mathrm{C}$ & -8.5788240 & 0.7580470 & 3.7571500 \\
\hline 41 & $\mathrm{C}$ & -8.9519520 & 0.8235720 & 5.0973200 \\
\hline 42 & $\mathrm{C}$ & -4.2957600 & 0.1488000 & 0.0543490 \\
\hline 43 & $\mathrm{C}$ & -4.1499090 & -0.0309260 & 2.5546090 \\
\hline 44 & $\mathrm{C}$ & -1.1584610 & -0.2690310 & 0.4530290 \\
\hline 45 & $\mathrm{C}$ & -5.0427960 & 1.2716570 & -1.7282770 \\
\hline 46 & $\mathrm{C}$ & -7.5953070 & 3.1255190 & -4.6058010 \\
\hline 47 & $\mathrm{C}$ & -5.9062490 & 1.9381670 & -2.7396100 \\
\hline 48 & $\mathrm{C}$ & 0.2300180 & -0.3507390 & 0.5346890 \\
\hline 49 & $\mathrm{C}$ & 12.9402630 & -1.0831720 & 1.3369450 \\
\hline 50 & $\mathrm{C}$ & -3.2429890 & 0.6104820 & -0.7180320 \\
\hline 51 & $\mathrm{C}$ & -9.2582740 & 2.9909430 & -1.3913130 \\
\hline 52 & $\mathrm{C}$ & -8.1506280 & 2.4340740 & -0.7365050 \\
\hline 53 & $\mathrm{C}$ & 11.5500380 & -0.9901900 & 1.2563970 \\
\hline 54 & $\mathrm{C}$ & 3.1399120 & -1.0241740 & -0.5611710 \\
\hline 55 & $\mathrm{C}$ & 8.7334300 & -1.3823640 & -0.2347940 \\
\hline 56 & $\mathrm{C}$ & -1.7810870 & 0.4852840 & -0.5574170 \\
\hline 57 & $\mathrm{C}$ & 13.7407880 & -0.4993460 & 0.3534530 \\
\hline 58 & $\mathrm{C}$ & 1.0564180 & 0.3183150 & -0.3796530 \\
\hline 59 & $\mathrm{C}$ & -6.2595590 & 3.2899010 & -2.5778660 \\
\hline 60 & $\mathrm{C}$ & -6.9967650 & 3.8926160 & -3.6061800 \\
\hline 61 & $\mathrm{C}$ & 10.9360880 & -0.3130110 & 0.1916160 \\
\hline 62 & $\mathrm{C}$ & -0.9497380 & 1.1548860 & -1.4752530 \\
\hline 63 & $\mathrm{C}$ & 2.5474590 & 0.2229210 & -0.2897460 \\
\hline
\end{tabular}




\begin{tabular}{|c|c|c|c|c|}
\hline 64 & $\mathrm{C}$ & 9.4417300 & -0.2163620 & 0.1048450 \\
\hline 65 & $\mathrm{C}$ & -7.1291130 & 3.3801990 & -0.1316500 \\
\hline 66 & $\mathrm{C}$ & 13.1420600 & 0.1775110 & -0.7107090 \\
\hline 67 & $\mathrm{C}$ & 0.4358520 & 1.0758570 & -1.3855100 \\
\hline 68 & $\mathrm{C}$ & 11.7516910 & 0.2693250 & -0.7906200 \\
\hline 69 & $\mathrm{C}$ & -6.1067690 & 3.9569570 & -1.2278810 \\
\hline 70 & $\mathrm{C}$ & 3.2582570 & 1.3867520 & 0.0566720 \\
\hline 71 & $\mathrm{C}$ & 8.8529630 & 1.0328420 & 0.3684170 \\
\hline 72 & $\mathrm{C}$ & 2.6397820 & 2.6430730 & 0.4106840 \\
\hline 73 & $\mathrm{C}$ & 9.5895130 & 2.2255350 & 0.7175720 \\
\hline 74 & $\mathrm{C}$ & 4.8875880 & 2.7982250 & 0.5319730 \\
\hline 75 & $\mathrm{C}$ & 7.3720150 & 2.6418750 & 0.6716430 \\
\hline 76 & $\mathrm{C}$ & 3.6438330 & 3.5130130 & 0.7043740 \\
\hline 77 & $\mathrm{C}$ & 8.6776960 & 3.2199740 & 0.8932740 \\
\hline 78 & $\mathrm{C}$ & 6.1605750 & 3.3500190 & 0.7659100 \\
\hline 79 & $\mathrm{C}$ & 6.2288130 & 4.7968190 & 1.1518280 \\
\hline 80 & $\mathrm{C}$ & 6.6216080 & 5.1789520 & 2.4441010 \\
\hline 81 & $\mathrm{C}$ & 5.8993890 & 5.8016750 & 0.2287420 \\
\hline 82 & $\mathrm{C}$ & 6.6848520 & 6.5260430 & 2.8029270 \\
\hline 83 & $\mathrm{C}$ & 5.9627860 & 7.1490480 & 0.5864440 \\
\hline 84 & $\mathrm{C}$ & 6.3559250 & 7.5157690 & 1.8748300 \\
\hline 85 & $\mathrm{H}$ & -9.4728590 & -6.5575360 & 1.3409110 \\
\hline 86 & $\mathrm{H}$ & -7.4911270 & -6.0967190 & -0.0904330 \\
\hline 87 & $\mathrm{H}$ & -10.5852880 & -4.6901790 & 2.5483900 \\
\hline 88 & $\mathrm{H}$ & 5.5759020 & -8.5463930 & -2.3779340 \\
\hline 89 & $\mathrm{H}$ & 4.7554590 & -7.8174730 & -0.1447870 \\
\hline 90 & $\mathrm{H}$ & -6.6318060 & -3.7748690 & -0.3059950 \\
\hline 91 & $\mathrm{H}$ & 6.5144810 & -6.8639660 & -3.9532040 \\
\hline 92 & $\mathrm{H}$ & -9.7108740 & -2.3904100 & 2.3537780 \\
\hline 93 & $\mathrm{H}$ & 4.8752480 & -5.4285230 & 0.5033910 \\
\hline 94 & $\mathrm{H}$ & -4.0409010 & -3.9010240 & 2.3376440 \\
\hline 95 & $\mathrm{H}$ & -9.9209140 & -0.5388460 & -4.2283110 \\
\hline 96 & $\mathrm{H}$ & -7.8676980 & -2.2388190 & 6.1115470 \\
\hline 97 & $\mathrm{H}$ & -7.2072000 & -2.3603430 & 3.7324680 \\
\hline 98 & $\mathrm{H}$ & -8.0423060 & -0.1420580 & -5.4813070 \\
\hline 99 & $\mathrm{H}$ & -8.3287530 & -0.6449700 & -2.1749830 \\
\hline
\end{tabular}




\begin{tabular}{|c|c|c|c|c|}
\hline 100 & $\mathrm{H}$ & -4.2775600 & -2.5441840 & 0.2723630 \\
\hline 101 & $\mathrm{H}$ & -10.6203210 & 1.0226820 & -4.6405230 \\
\hline 102 & $\mathrm{H}$ & -8.8992870 & 1.2439480 & -6.1475310 \\
\hline 103 & $\mathrm{H}$ & -3.8483380 & -2.7741550 & 4.5482160 \\
\hline 104 & $\mathrm{H}$ & 3.1444080 & -4.1973280 & -1.5129300 \\
\hline 105 & $\mathrm{H}$ & 8.4292640 & -4.5789580 & -1.0497570 \\
\hline 106 & $\mathrm{H}$ & 6.6302710 & -4.4772120 & -3.2964150 \\
\hline 107 & $\mathrm{H}$ & -6.2663600 & 0.1201910 & -3.8106750 \\
\hline 108 & $\mathrm{H}$ & -8.9920700 & -0.2048200 & 6.9936560 \\
\hline 109 & $\mathrm{H}$ & 1.3446650 & -2.2395300 & -1.1519140 \\
\hline 110 & $\mathrm{H}$ & 10.4093970 & -2.8597380 & -0.4875620 \\
\hline 111 & $\mathrm{H}$ & -1.7520640 & -0.8007390 & 1.1855160 \\
\hline 112 & $\mathrm{H}$ & -10.6642520 & 2.8491480 & -3.0075060 \\
\hline 113 & $\mathrm{H}$ & 13.3973240 & -1.6091530 & 2.1711840 \\
\hline 114 & $\mathrm{H}$ & 0.6830750 & -0.9401560 & 1.3274420 \\
\hline 115 & $\mathrm{H}$ & -3.9236550 & -0.2914930 & 4.6781710 \\
\hline 116 & $\mathrm{H}$ & 10.9289340 & -1.4416060 & 2.0252580 \\
\hline 117 & $\mathrm{H}$ & -8.7616320 & 1.5929880 & 3.0884090 \\
\hline 118 & $\mathrm{H}$ & -9.4369470 & 1.7176970 & 5.4793250 \\
\hline 119 & $\mathrm{H}$ & -8.2879310 & 3.6001930 & -5.2983410 \\
\hline 120 & $\mathrm{H}$ & 14.8234030 & -0.5712730 & 0.4158350 \\
\hline 121 & $\mathrm{H}$ & -4.1764580 & 1.0528370 & 2.6097090 \\
\hline 122 & $\mathrm{H}$ & -9.5118020 & 4.0303000 & -1.1928860 \\
\hline 123 & $\mathrm{H}$ & -6.5655020 & 2.8913940 & 0.6623980 \\
\hline 124 & $\mathrm{H}$ & -1.4148810 & 1.7335470 & -2.2653340 \\
\hline 125 & $\mathrm{H}$ & -7.2230040 & 4.9550870 & -3.5443610 \\
\hline 126 & $\mathrm{H}$ & 13.7569370 & 0.6321340 & -1.4831990 \\
\hline 127 & $\mathrm{H}$ & -7.6495990 & 4.2312640 & 0.3215020 \\
\hline 128 & $\mathrm{H}$ & 1.0511660 & 1.5992360 & -2.1125810 \\
\hline 129 & $\mathrm{H}$ & -5.0903260 & 3.8315560 & -0.8416590 \\
\hline 130 & $\mathrm{H}$ & 11.2878390 & 0.7916860 & -1.6228530 \\
\hline 131 & $\mathrm{H}$ & -6.2795710 & 5.0329380 & -1.3340790 \\
\hline 132 & $\mathrm{H}$ & 1.5755570 & 2.8244920 & 0.4451650 \\
\hline 133 & $\mathrm{H}$ & 10.6636920 & 2.2894570 & 0.8125200 \\
\hline 134 & $\mathrm{H}$ & 3.5545390 & 4.5404610 & 1.0254830 \\
\hline 135 & $\mathrm{H}$ & 6.8718010 & 4.4102820 & 3.1700210 \\
\hline
\end{tabular}




\begin{tabular}{rrrrr}
136 & $\mathrm{H}$ & 8.8679140 & 4.2521240 & 1.1486570 \\
137 & $\mathrm{H}$ & 5.5979780 & 5.5193120 & -0.7761900 \\
138 & $\mathrm{H}$ & 6.9872410 & 6.8011970 & 3.8100750 \\
139 & $\mathrm{H}$ & 5.7080990 & 7.9124040 & -0.1442330 \\
140 & $\mathrm{H}$ & 6.4050340 & 8.5650280 & 2.1536760 \\
141 & $\mathrm{~N}$ & -7.1140590 & -1.1622320 & -0.2945830 \\
142 & $\mathrm{~N}$ & -7.0475200 & 0.6281610 & 1.2629070 \\
143 & $\mathrm{~N}$ & -5.4640480 & 0.5853540 & -0.5913620 \\
144 & $\mathrm{~N}$ & 4.4850740 & -1.3131570 & -0.4912450 \\
145 & $\mathrm{~N}$ & 7.3667110 & -1.4853430 & -0.3700630 \\
146 & $\mathrm{~N}$ & -3.7359400 & 1.3033950 & -1.8053810 \\
147 & $\mathrm{~N}$ & 4.6283210 & 1.5026320 & 0.1432910 \\
148 & $\mathrm{~N}$ & 7.5037600 & 1.3092000 & 0.3492290 \\
149 & $\mathrm{Zn}$ & 5.9951420 & 0.0039300 & -0.0939250 \\
\hline
\end{tabular}

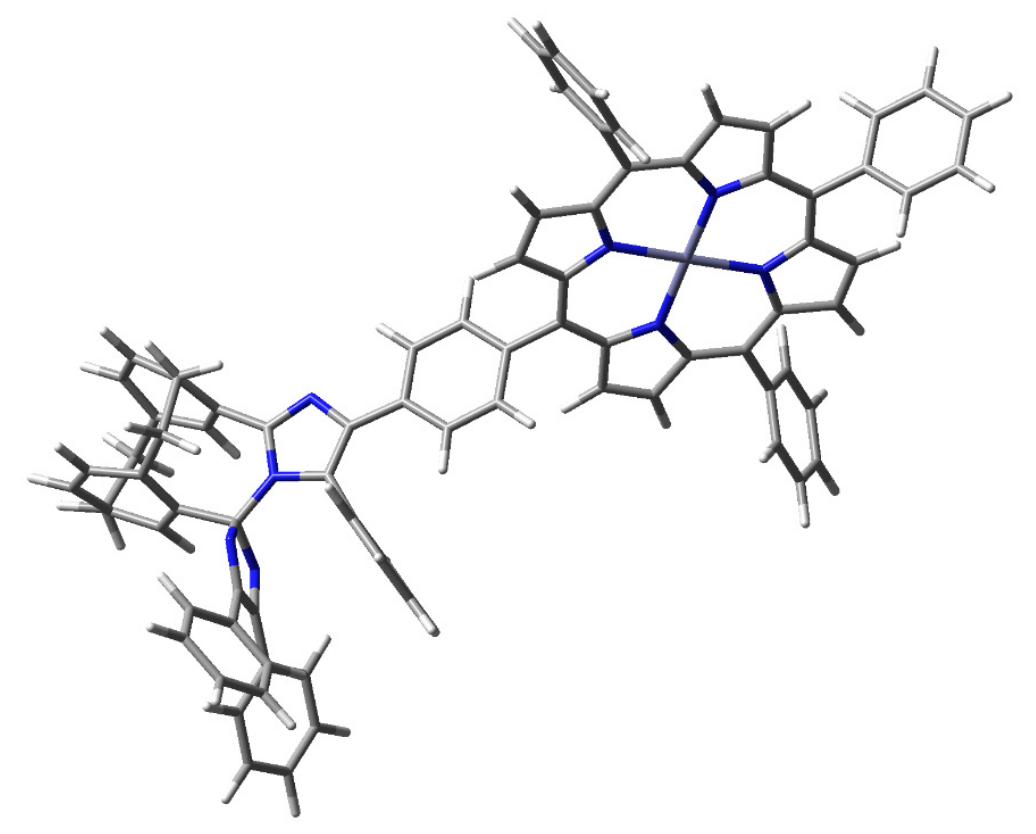

Figure S17. Optimized structure of $\mathbf{1}$. 
SCF Done: E(RB3LYP)

Zero-point correction

Thermal correction to Energy

Thermal correction to Enthalpy

Thermal correction to Gibbs Free Energy

Sum of electronic and zero-point Energies

Sum of electronic and thermal Energies

Sum of electronic and thermal Enthalpies

Sum of electronic and thermal Free Energies

Low frequencies ---

Low frequencies ---

$-2.139$

$6.4318 \quad 6.5493$ $=\quad-5450.39753145$

A.U.

$=1.175534$ (Hartree/Particle)

$=\quad 1.246499$

$=\quad 1.247443$

$=\quad 1.059808$

$=\quad-5450.696226$

$=\quad-5450.625262$

$=\quad-5450.624318$

$=$

$-5450.811953$

$\begin{array}{llll}-0.0042 & -0.0019 & 0.0007 & 1.2306\end{array}$

8.3539 


\section{References}

S1. Rehm, D.; Weller, A. Israel J. Chem. 1970, 8, 159.

S2. Felton, R. H.; Linshits, H., J. Am. Chem. Soc. 1966, 88, 1113.

S3. Mutoh, K.; Nakano, E., Abe, J. J. Phys. Chem. A, 2012, 116, 6792.

S4. Frisch, M. J.; Trucks, G. W.; Schlegel, H. B.; Scuseria, G. E.; Robb, M. A.; Cheeseman, J. R.; Scalmani, G.; Barone, V.; Mennucci, B.; Petersson, G. A.; Nakatsuji, H.; Caricato, M.; Li, X.; Hratchian, H. P.; Izmaylov, A. F.; Bloino, J.; Zheng, G.; Sonnenberg, J. L.; Hada, M.; Ehara, M.; Toyota, K.; Fukuda, R.; Hasegawa, J.; Ishida, M.; Nakajima, T.; Honda, Y.; Kitao, O.; Nakai, H.; Vreven, T.; Montgomery, J. A., Jr.; Peralta, J. E.; Ogliaro, F.; Bearpark, M.; Heyd, J. J.; Brothers, E.; Kudin, K. N.; Staroverov, V. N.; Kobayashi, R.; Normand, J.; Raghavachari, K.; Rendell, A.; Burant, J. C.; Iyengar, S. S.; Tomasi, J.; Cossi, M.; Rega, N.; Millam, N. J.; Klene, M.; Knox, J. E.; Cross, J. B.; Bakken, V.; Adamo, C.; Jaramillo, J.; Gomperts, R.; Stratmann, R. E.; Yazyev, O.; Austin, A. J.; Cammi, R.; Pomelli, C.; Ochterski, J. W.; Martin, R. L.; Morokuma, K.; Zakrzewski, V. G.; Voth, G. A.; Salvador, P.; Dannenberg, J. J.; S. 50 Dapprich, S.; Daniels, A. D.; Farkas, Ö.; Foresman, J. B.; Ortiz, J. V.; Cioslowski, J.; Fox, D. J. Gaussian 09, Revision D.01; Gaussian, Inc.: Wallingford CT, 2009. 